\title{
Vers une écriture collective transformative au primaire : interventions enseignantes et design technologique
}

Stéphane ALLAIRE, Pascale THÉRIAULT et Vincent

GAGNON (UNIVERSITÉ DU QUÉBEC À CHICOUTIMI, Saguenay), Thérèse LAFERrIÈre, Christine hamel et Pier-Ann BoUtin, (UNIVERSITÉ LAVAL, Québec)

Godelieve DEBEURME, (UNIVERSITÉ DE SHERBROOKE, Sherbrooke), Chercheurs et étudiants-chercheurs associés au Centre de recherche et $d^{\prime}$ 'intervention sur la réussite scolaire (CRIRES)

口 RÉSUMÉ • Ce texte rend compte d'une étude qui a documenté la façon dont les interventions enseignantes et les affordances d'un outil d'écriture collective, le Knowledge Forum, peuvent se compléter pour amener des élèves du primaire à prendre part à une écriture davantage transformative. Le cadre théorique s'appuie entre autres sur les processus et les stratégies d'écriture. La méthode a combiné l'observation d'interventions effectuées en classe, des entrevues ainsi que l'observation des écrits sur le forum électronique. Les résultats mettent en lumière notamment une démarche d'écriture qui amène les élèves à dépasser une énonciation spontanée de leurs connaissances.

MOTS-CLÉS • Écriture collective, stratégies d'écriture, classe en réseau, TIC

ABSTRACT - This paper reports results of a study that documented the way teachers' intervention and affordances of a collective writing tool, Knowledge Forum, can combine to engage primary pupils in transformative writing. Theoretical frameword is based on writing processes and strategies. Method combines observation of teachers' intervention, interviews and observation of notes written in the electronic forum. Results reveal how it is possible to bring students over a knowledge telling approach to writing.

- KEYWORDS • Collective writing, writing strategies, networked classroom, ICT

Stéphane ALLAIRE, Pascale THÉRIAULT, Vincent GAGNON, Thérèse LAFERRIÈRE, Chrisitne HAMEL, Pier-Ann BOUTIN, Godelieve DEBEURME

Vers une écriture collective transformative au primaire : interventions enseignan-

tes et design technologique

Sticef, vol. 20, 2013, en ligne sur <www.sticef.org> 
Stéphane ALLAIRE, Pascale THÉRIAULT, Vincent GAGNON, Thérèse LAFERRIÈRE, Christine HAMEL, Pier-Ann BOUTIN, Godelieve DEBEURME.

\section{Problématique}

Les impératifs d'une société dont le fonctionnement s'aligne sur la primauté du savoir exercent de plus en plus de pression sur les systèmes éducatifs (Bereiter, 2002), (Schleicher, 2012). Une implication courante d'une telle société veut qu'un accent soit mis sur la démocratisation des connaissances, notamment par un accès facilité à ces dernières. Une autre implication, plus ambitieuse celle-là, concerne la pertinence et la nécessité de développer des pratiques de coélaboration, voire de création de connaissances et ce, dès l'éducation primaire. Pour Bereiter et Scardamalia (Bereiter et Scardamalia, 2005), en l'absence de procédure explicite pour y parvenir, il est souhaitable d'adopter une approche développementale et d'y travailler le plus tôt possible, de sorte à exercer et à raffiner de façon progressive les aptitudes des élèves et des étudiants, tout au long de leur cheminement scolaire. Peu importe qu'il s'agisse de l'une ou l'autre implication, les technologies de l'information et de la communication (TIC) sont de plus en plus envisagées comme un catalyseur de la société du savoir (UNESCO, 2005).

Des changements s'opèrent au niveau d'orientations-cadres, dont les programmes de formation font partie. Par exemple, en 2011, 1'UNESCO a publié la mise à jour d'un référentiel de compétences destiné aux enseignants (UNESCO, 2011). La création de connaissances y est bien mise en valeur. En ce qui concerne les élèves, l'Assessment and Teaching of $21^{\text {st }}$ Century Skills fait de la créativité et de l'innovation une des dix compétences du $21^{\mathrm{e}}$ siècle. Au Québec, en dépit du fait qu'ils ne soient pas tous soumis à une évaluation systématique et formelle, de tels éléments se manifestent notamment à travers des compétences transversales telles « Résoudre des problèmes», «Exercer son jugement critique » ainsi que «Mettre en œuvre sa pensée créatrice » $(\mathrm{MEQ}, 2001)$. Ces tangentes générales insufflent un vent de changement aux pratiques de classe.

L'écriture, socle des apprentissages scolaires avec la lecture, est bien connue pour sa fonction de communication d'idées. Une autre fonction, parfois moins connue et moins mise à contribution de façon systématique en classe, est sa fonction épistémique (Blaser, 2007). Elle vise alors l'appropriation, voire la création de connaissances. Des travaux, notamment ceux de Bereiter et Scardamalia (Bereiter et Scardamalia, 1987) à propos de l'expertise en écriture, ont montré son potentiel transformatif. Cela signifie que le fait de prendre part à l'acte d'écrire, en soi, peut amener un individu à se forger des idées qu'il n'avait pas auparavant. Une 
telle dynamique s'inscrit dans la prolongation des travaux de Vygotsky (Vygotsky, 1978) à propos de l'interinfluence entre la pensée et le langage.

Or, l'adoption d'une écriture transformative ne va pas de soi, en particulier chez les scripteurs novices, auxquels appartiennent les élèves du primaire. Ceux-ci ont plutôt tendance à rédiger au fil de leurs idées, jusqu'à ce qu'ils n'en aient plus. C'est à ce moment alors, qu'à leurs yeux, le texte est complété (Bereiter et Scardamalia, 1987). Un processus de récitation de connaissances (knowledge telling) a aussi été observé par Allaire et al. (Allaire et al., 2011) et (Allaire et al., 2013) lors de l'usage de médias sociaux, en particulier du blogue. Si un tel processus est efficace du point de vue de la forme scolaire traditionnelle et de la gestion du temps (c'està-dire compléter la tâche assignée en un minimum de temps), il se distingue par ailleurs du processus adopté par les scripteurs avancés, qui, eux, prennent davantage de temps pour peaufiner, retravailler l'écrit afin qu'il corresponde le mieux possible à l'intention ciblée. L'effort requis par un tel accomplissement serait favorable à une transformation des idées, en raison des allers-retours itératifs entre des espaces " contenu » et « rhétorique » (Bereiter et Scardamalia, 1987).

Dans le cadre d'une recherche financée par le «Fonds de recherche du Québec sur la société et la culture » (FRQSC), nous travaillons notamment à la mise en place et au raffinement de pratiques d'écriture axées sur l'amélioration des idées. De nature transformative, ces pratiques sont soutenues par un forum électronique de coélaboration de connaissances, le Knowledge Forum. Ce texte présente la façon dont des interventions d'enseignantes du primaire se sont combinées aux affordances de l'outil retenu afin d'amener les élèves à prendre part à une écriture pouvant être qualifiée de transformative, et qui s'inscrit dans la perspective des exigences d'une société du savoir.

\section{Cadre théorique}

Les travaux issus de la théorie de l'activité (Vygotsky, 1978), (Engeström, 1987) soutiennent que toute activité humaine se déploie par l'entremise d'outils, qu'ils soient symboliques et/ou conceptuels, mécaniques ou numériques. Ainsi, les interventions effectuées par les individus qui poursuivent un objet, un but spécifique prennent-elles forme à l'aide - et à travers - de tels outils.

Quant aux usages, le concept de genèse instrumentale de Rabardel (Rabardel, 1995) fournit un rationnel qui nous apparait pertinent pour 
Stéphane ALLAIRE, Pascale THÉRIAULT, Vincent GAGNON, Thérèse LAFERRIÈre, Christine HAMEL, Pier-Ann BOUTIN, Godelieve DEBEURME.

conceptualiser l'appropriation et l'utilisation d'un outil donné, en conjonction avec les actions d'un individu. Deux mouvements sont en jeu selon l'auteur. D'une part, sur la base de son identité et de ce qu'il accomplit, l'individu attribue une ou des fonctions aux outils qu'il utilise. Il peut en quelque sorte les modeler, les adapter à ce qu'il souhaite accomplir. Il s'agit du mouvement d'instrumentalisation et c'est lui qui entre en jeu lorsque par exemple, une personne utilise, en l'absence de tournevis, un couteau pour extraire une vis d'un meuble. L'outil n'est pas conçu pour effectuer une telle tâche; on lui attribue un usage. D'autre part, en fonction de ses propriétés et caractéristiques, tout outil induit aussi une façon de l'utiliser auprès d'un individu. Pour illustrer ce mouvement d'instrumentation, on peut référer à l'exemple classique de la chaise qui, par sa solidité apparente et la hauteur de sa surface plane, invite un individu à s'y asseoir. C'est dire qu'un outil peut suggérer des actions, voire des interventions à un individu (Allaire, 2006).

L'interaction entre les mouvements d'instrumentalisation et d'instrumentation engendre donc des potentiels d'action - des affordances (Gaver, 1991), (Gibson, 1979) - qui alimentent la façon dont une activité est mise en œuvre. Cette interaction est importante à considérer, en particulier compte tenu de la dimension téléologique de l'enseignement, c'est-à-dire de la poursuite délibérée d'objets d'apprentissage. Dans notre contexte, il s'agit de documenter la façon dont s'orchestrent les interventions d'enseignantes du primaire alors qu'un outil d'écriture collective, le Knowledge Forum, est utilisé en classe dans l'optique de favoriser une écriture transformative chez les élèves. Le Knowledge Forum est un forum électronique axé sur la résolution de problèmes authentiques en collaboration. Un principe sous-jacent important est à l'effet que c'est par l'intermédiaire des interactions écrites entre participants que les idées prennent forme progressivement et que la communauté peut parvenir à mieux comprendre le problème sur lequel elle se penche. Particulièrement, les participants élaborent des notes à partir des propos des autres afin d'améliorer cette compréhension. Les notes sont reliées graphiquement pour permettre de suivre la progression du discours écrit collectif.

Par ailleurs, il est reconnu que les scripteurs avancés mobilisent des stratégies en regard des trois principaux processus que sont la planification, la mise en texte ainsi que la révision et la correction (Hayes et Flower, 1980), (Hayes, 1995). Ces processus se déploient en de nombreuses stratégies (MEQ, 2001) qui sont énoncées au Tableau. Ces stratégies orienteront le cadre d'analyse des interventions enseignantes. 


\begin{tabular}{|c|c|}
\hline $\begin{array}{l}\text { Processus } \\
\text { d'écriture }\end{array}$ & Stratégies d'écriture \\
\hline \multirow{7}{*}{ Planification } & Se rappeler les expériences d'écriture déjà vécues. \\
\hline & $\begin{array}{l}\text { Utiliser un déclencheur pour stimuler son imaginaire (ex. } \\
\text { : œuvres d'art, illustrations, objets). }\end{array}$ \\
\hline & $\begin{array}{l}\text { Préciser son intention d'écriture et la garder constamment } \\
\text { à l'esprit. }\end{array}$ \\
\hline & Penser au destinataire du texte à produire. \\
\hline & $\begin{array}{l}\text { Évoquer un contenu possible (exploration et choix des } \\
\text { idées). }\end{array}$ \\
\hline & Anticiper le déroulement ou l'organisation du texte. \\
\hline & $\begin{array}{l}\text { Dresser une carte d'exploration, un croquis, un schéma, } \\
\text { un plan sommaire ou toute autre forme de support perti- } \\
\text { nent. }\end{array}$ \\
\hline \multirow{4}{*}{ Mise en texte } & Ajouter au fur et à mesure les idées qui viennent. \\
\hline & $\begin{array}{l}\text { Rédiger une première version à partir des idées formulées } \\
\text { mentalement. }\end{array}$ \\
\hline & $\begin{array}{l}\text { Retourner aux données du projet d'écriture ou à un sup- } \\
\text { port externe. }\end{array}$ \\
\hline & Relire la partie rédigée pour enchainer la suite. \\
\hline \multirow{16}{*}{$\begin{array}{l}\text { Révision, cor- } \\
\text { rection, auto- } \\
\text { évaluation }\end{array}$} & $\begin{array}{l}\text { Se demander si ce qui est écrit correspond bien à ce que } \\
\text { l'on veut dire. }\end{array}$ \\
\hline & Repérer les passages à reformuler. \\
\hline & Réfléchir à des modifications possibles. \\
\hline & $\begin{array}{l}\text { Lire oralement son texte à une ou plusieurs personnes, ou } \\
\text { leur demander de le lire, afin d'obtenir des suggestions } \\
\text { d'amélioration (structure, contenu, langue). }\end{array}$ \\
\hline & $\begin{array}{l}\text { Choisir, parmi les suggestions obtenues, celles qui sem- } \\
\text { blent le plus appropriées. }\end{array}$ \\
\hline & $\begin{array}{l}\text { Modifier le texte en recourant aux opérations syntaxiques } \\
\text { (ajout, effacement, déplacement, remplacement de grou- } \\
\text { pes de mots ou de phrases). }\end{array}$ \\
\hline & Relire son texte plus d'une fois. \\
\hline & $\begin{array}{l}\text { Inscrire, s'il y a lieu, des marques, des traces ou des sym- } \\
\text { boles pouvant servir de rappel ou d'aide-mémoire. }\end{array}$ \\
\hline & $\begin{array}{l}\text { Recourir à une procédure de correction ou } \\
\text { d'autocorrection. }\end{array}$ \\
\hline & Consulter les outils de référence disponibles. \\
\hline & Recourir à un autre élève ou à un adulte. \\
\hline & $\begin{array}{l}\text { Utiliser les ressources d'un traitement de texte et d'un cor- } \\
\text { recteur intégré. }\end{array}$ \\
\hline & Décrire ou expliquer la démarche suivie. \\
\hline & Vérifier l'atteinte de l'intention d'écriture. \\
\hline & Se prononcer sur l'efficacité des stratégies retenues. \\
\hline & S'autoévaluer comme scripteur. \\
\hline
\end{tabular}

Tableau 1 • Processus et stratégies d'écriture (MEQ, 2001)

Pour ce qui est de l'articulation des interventions des enseignants avec l'outil d'écriture, nous retenons les principales affordances perceptibles (Gaver, 1991) du Knowledge Forum, c'est-à-dire celles dont le design a été 


\section{Stéphane ALLAIRE, Pascale THÉRIAULT, Vincent GAGNON, Thérèse LAFERRIÈRE, Christine HAMEL, Pier-Ann BOUTIN, Godelieve DEBEURME.}

conçu intentionnellement pour instrumenter des interventions de coélaboration, voire de création de connaissances. Cette dynamique réfère à un processus collectif où les membres d'une communauté d'apprenants cherchent délibérément, en mettant à contribution l'écrit, à améliorer des idées (Bereiter et Scardamalia, 2003). Ces idées se rapportent à une question ou à un problème donné et elles ont de la valeur à des fins de compréhension, d'explication ou de résolution de problème complexe. Faute d'espace, nous n'entrons pas dans une description exhaustive de chacune des affordances - voir le chapitre 4 de (Allaire et Lusignan, 2011) pour plus d'informations à cet effet, ou encore la version originale anglaise des principes de (Scardamalia et Bereiter, 2006). Nous porterons principalement attention à celles du Tableau 2.

\begin{tabular}{|l|l|}
\hline $\begin{array}{l}\text { Affordances percep- } \\
\text { tibles }\end{array}$ & Instrumentation \\
\hline Nouvelle note & $\begin{array}{l}\text { Amorcer une thématique inexplorée jusqu'à présent par la } \\
\text { communauté }\end{array}$ \\
\hline Élaboration & Enrichir le contenu d'une note existante \\
\hline Annotation & $\begin{array}{l}\text { Commenter le contenu d'une note de façon plus personna- } \\
\text { lisée }\end{array}$ \\
\hline $\begin{array}{l}\text { Échafaudage (étaya- } \\
\text { ge) }\end{array}$ & $\begin{array}{l}\text { Annoncer le type de contenu qu'on désire rédiger à } \\
\text { l'intérieur d'une note }\end{array}$ \\
\hline Champ « Problème » & Nommer l'objet abordé \\
\hline Champ « Mot-clé » & $\begin{array}{l}\text { Identifier les mots ou concepts importants du contenu } \\
\text { d'une note }\end{array}$ \\
\hline $\begin{array}{l}\text { Note « Élever le } \\
\text { propos » }\end{array}$ & $\begin{array}{l}\text { Regrouper plusieurs notes pour en rédiger l'équivalent } \\
\text { d'une synthèse, voire suggérer une avancée }\end{array}$ \\
\hline Citation & $\begin{array}{l}\text { Référer explicitement à un contenu élaboré antérieurement } \\
\text { par un ou plusieurs membres de la communauté }\end{array}$ \\
\hline Publication & $\begin{array}{l}\text { Attester de la qualité du contenu d'une perspective (ensem- } \\
\text { ble de notes) en suggérant de rendre ce contenu accessible à } \\
\text { d'autres personnes que les membres de la communauté qui } \\
\text { les a produites }\end{array}$ \\
\hline Perspective & Créer un nouvel espace numérique de coélaboration \\
\hline $\begin{array}{l}\text { Idées prometteuses } \\
\text { Sélectionner les idées qui, du point de vue des membres de } \\
\text { la communauté, méritent d'être approfondies (Boutin, } \\
\text { 2013) }\end{array}$ \\
\hline $\begin{array}{l}\text { Organisation spatiale } \\
\text { et visuelle de l'espace } \\
\text { collectif d'écriture }\end{array}$ & ter leur lecture et compréhension \\
\hline
\end{tabular}

Tableau 2 • Affordances perceptibles du Knowledge Forum et leur instrumentation

Nous décrivons maintenant la méthode retenue dans le cadre de l'étude. 


\section{Méthode}

\subsection{Participants}

La recherche est en cours depuis deux ans et une quinzaine de classes du primaire provenant de cinq régions administratives du Québec y ont participé jusqu'à présent. Dans ce texte, nous nous concentrons sur les données collectées auprès de deux enseignantes et leurs élèves de troisième cycle du primaire (10-11 ans) provenant d'un milieu socioéconomique défavorisé. L'objectif du texte étant d'illustrer comment des interventions en écriture peuvent tirer profit d'une technologie collective d'écriture, notre choix des participants s'est ici concentré sur des enseignantes qui avaient une pratique de type étayé (Allaire et al., 2013), c'est-à-dire offrant un soutien aux élèves dans une optique de développement progressif de leur autonomie en écriture ${ }^{1}$.

Les deux enseignantes ont une classe en réseau dont le fonctionnement et la dynamique d'enseignement-apprentissage s'inspirent des principes promus par le modèle de "L'école en réseau " (Laferrière et al., 2011). En particulier, les élèves d'une classe donnée travaillent périodiquement avec des élèves d'une ou de plusieurs autres classes à partir du Knowledge Forum et de la webconférence (VIA) à des fins d'enrichissement de l'environnement d'apprentissage et de la réussite scolaire. Les deux enseignants possédaient une longue expérience du modèle ( 7 et 8 ans).

\subsection{Déroulement de l'expérimentation}

Pour la situation d'écriture qui fait ici l'objet d'analyse, les classes des deux enseignantes ont travaillé ensemble. Plus précisément, elles ont travaillé l'apprentissage de l'écriture à travers un contenu d'histoire (univers social) : le mode de vie au Moyen Âge. Il s'agissait pour les deux communautés d'apprenants de chercher à mieux comprendre cette société. Le travail s'est échelonné sur plusieurs semaines à l'automne 2011. Dans chaque classe participante, quelques ordinateurs étaient à la disposition des élèves. Ces derniers ont donc travaillé sur le forum électronique à tour de rôle. Précisons aussi que la réalisation de cette situation d'écriture relève des intentions pédagogiques initiales des enseignantes et non d'un devis imposé par l'équipe de recherche.

Tel que mentionné précédemment, Knowledge Forum a été l'outil d'écriture utilisé. Son interface neuronale a été privilégiée (Figure 1) car elle offre davantage de souplesse pédagogique. Par exemple, les notes 
Stéphane ALLAIRE, Pascale THÉRIAULT, Vincent GAGNON, Thérèse LAFERRIÈRE, Christine HAMEL, Pier-Ann BOUTIN, Godelieve DEBEURME.

(icônes) peuvent être déplacées pour former des regroupements sémantiques logiques.

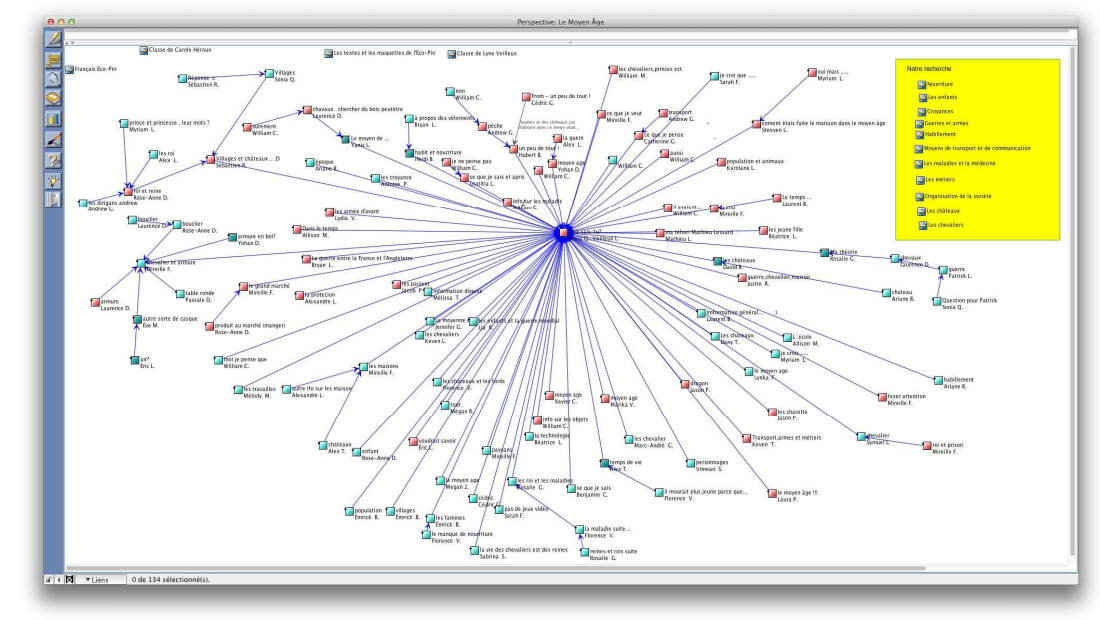

Figure $1 \bullet$ Interface neuronale du Knowledge Forum

Au premier regard, un tel type d'interface se distingue de celui des forums électroniques plus classiques (par arborescence) par la non-linéarité de la présentation des messages, appelés notes. La couleur turquoise des icônes indique une note non lue par un participant alors que le rouge indique qu'elle l'a été. Lorsqu'une note est révisée, elle redevient turquoise, mais d'un ton plus foncé.

Dans le cadre de la situation d'écriture retenue, le Knowledge Forum a été utilisé en conjonction avec des échanges et du travail en face à face. Parfois, ce travail a été effectué en groupe, en équipe locale d'élèves, entre des élèves de classes différentes (par webconférence) ou de façon individuelle.

Par ailleurs, considérant la dimension de changement de pratique ciblée dans le cadre général de la recherche-action, les chercheurs se sont impliqués à titre d'intervenants lors de son déroulement. Ils l'ont fait de deux principales façons. D'une part, ils ont partagé des données descriptives aux enseignantes. Ces données mettaient en lumière les liens entre leurs interventions et les stratégies d'écriture et elles ont donné lieu à des échanges qui ont permis d'effectuer des ajustements de pratique. D'autre part, l'équipe de recherche a présenté la pertinence et le fonctionnement de l'affordance des idées prometteuses. 


\subsection{Collecte et analyse de données}

La collecte de données a été effectuée selon trois principales modalités. Des observations directes des enseignantes en action pendant le déroulement de la situation d'écriture ont été effectuées en différé, à partir d'enregistrements en webconférence. Il s'agissait d'observer le soutien offert par les enseignantes aux élèves en regard des processus d'écriture (planification, mise en texte ainsi que révision et correction), que ce soit en face à face ou sur le forum électronique. Ensuite, des entrevues semidirigées ont eu lieu avec les enseignantes pour compléter les observations directes ainsi que pour saisir ou valider les intentions à la base de leurs interventions. Enfin, les affordances du Knowledge Forum utilisées et les écrits rédigés par les élèves et les enseignantes ont été considérés.

Pour ce qui est de la méthode d'analyse, les interventions des enseignantes ont été catégorisées à partir des stratégies d'écriture énoncées dans le " Programme de formation de l'école québécoise » (MEQ, 2001), (voir Tableau 1). Le cas échéant, les interventions ont aussi été associées aux affordances du forum électronique. Enfin, des écrits d'élèves ont été analysés sous l'angle de l'ajout d'idées par rapport à celles formulées en début de situation d'écriture. Pour ce faire, chaque idée différente a été codifiée selon qu'elle se retrouvait sur le forum électronique et/ou dans le texte individuel d'un élève. L'analyse a aussi porté sur la provenance des idées, c'est-à-dire les notes rédigées par chaque élève ou celles de leurs camarades.

\section{Présentation des résultats}

Globalement, la situation d'écriture documentée a été orchestrée selon cinq phases, dont les trois premières ont mis à profit le Knowledge Forum. Nous débutons par présenter la description du déroulement pour chacune de ces phases. À ce niveau, le lecteur ne devrait pas être surpris par la tangente ethnographique du regard posé. Ce regard a permis de dégager un modèle d'écriture en cinq phases. Ensuite, nous présentons des résultats en lien avec les écrits individuels des élèves.

\subsection{Phase 1 (durée approximative : une semaine)}

La phase 1 a consisté en l'amorce de la situation d'écriture. Les enseignantes ont ancré l'intention d'écriture dans un contexte de résolution de problème en expliquant aux élèves qu'il s'agissait, dans un premier temps, de s'engager collectivement dans une recherche d'informations pour mieux comprendre le fonctionnement de la société au Moyen Âge. Ulti- 
Stéphane ALLAIRE, Pascale THÉRIAULT, Vincent GAGNON, Thérèse LAFERRIÈre, Christine HAMEL, Pier-Ann BOUTIN, Godelieve DEBEURME.

mement, les élèves auraient à produire un texte informatif individuel ainsi qu'une maquette en arts plastiques pour rendre compte d'une partie de cette compréhension. Par la webconférence réunissant les deux classes, une tempête d'idées a été effectuée à propos de ce que les élèves connaissaient du Moyen Âge, à titre d'élément déclencheur de l'écriture. Les élèves ont ensuite été invités à garder des traces écrites de ces premières idées, sur le forum électronique. En guise de rappel de cette intention d'écriture, les enseignantes ont créé une nouvelle perspective (entendre la nouvelle page dans l'espace collectif) intitulée " Moyen Âge » pour l'écriture collective. En outre, elles ont rédigé la première contribution, soit une note qui invitait les élèves à rédiger ce qu'ils connaissaient de la société de l'époque (Figure 2) dans cet espace numérique collectif. Les enseignantes en ont profité pour expliquer et modéliser l'utilisation de l'affordance d'échafaudage. Celle-ci a été présentée comme un appui à la clarification d'une intention d'écriture sous la forme d'une nouvelle contribution dans la perspective à développer. En l'occurrence ici, c'est l'échafaudage «J'ai besoin de comprendre » qui a été utilisé puisque le contenu de la note initiale des enseignantes consistait en la formulation du questionnement à la base de l'écriture.

\begin{tabular}{|l|l|l|l|l|}
\hline Note Auteurs Liens & Info & History \\
\hline Constructin d'une théorie
\end{tabular}

Figure 2 • Note d'amorce de la situation d'écriture rédigée par les enseignantes

Les élèves ont été invités à lire les notes de leurs camarades pour s'enquérir de leurs idées, pour ne pas les répéter (compte tenu de la dimension collective du forum électronique), voire ajouter des idées complémentaires, et ce en dépit du fait qu'à ce stade-ci, l'intention était surtout de permettre à la fois un rappel des connaissances et la formulation de conceptions spontanées sur la question, et d'en garder trace. Des idées complémentaires ont été associées de par l'affordance d'élaboration, 
qui permet de relier graphiquement une note à une autre. D'un point de vue collectif, elle a soutenu un enchainement logique des idées. Par exemple, à partir d'une note qui formulait une idée personnelle à l'effet que c'est le roi qui dirigeait la société à l'époque du Moyen Âge, un élève a élaboré en précisant que les princes et les princesses avaient aussi, selon lui, un certain pouvoir décisionnel.

Par ailleurs, bien que le Knowledge Forum ait été présenté comme un espace collectif d'écriture permettant d'améliorer la compréhension de quelque chose et que, par conséquent, la répétition d'un même contenu n'était pas souhaitable, les enseignantes ont expliqué aux élèves qu'en cas de telles répétitions, il était possible d'utiliser l'affordance d'organisation spatiale et visuelle pour regrouper des notes selon leur proximité sémantique. Une telle façon de faire se veut contributoire à la réorganisation de l'écriture collective.

\subsection{Phase 2 (durée approximative : deux à trois semaines)}

L'objectif de la seconde phase de la situation d'écriture était d'amener les élèves à dépasser les connaissances antérieures ou les conceptions spontanées énoncées lors de la première phase. Pour ce faire, les enseignantes, soutenues par un membre de l'équipe de recherche, ont d'abord demandé aux élèves d'utiliser l'affordance des idées prometteuses intégrée au Knowledge Forum. Ainsi, chaque élève a relu les notes rédigées jusque là et a identifié, par surlignement, les éléments qui lui semblaient importants d'approfondir pour parvenir à une compréhension plus étoffée du Moyen Âge (Figure 3). 
Stéphane ALLAIRE, Pascale THÉRIAULT, Vincent GAGNON, Thérèse LAFERRIÈRE, Christine HAMEL, Pier-Ann BOUTIN, Godelieve DEBEURME.

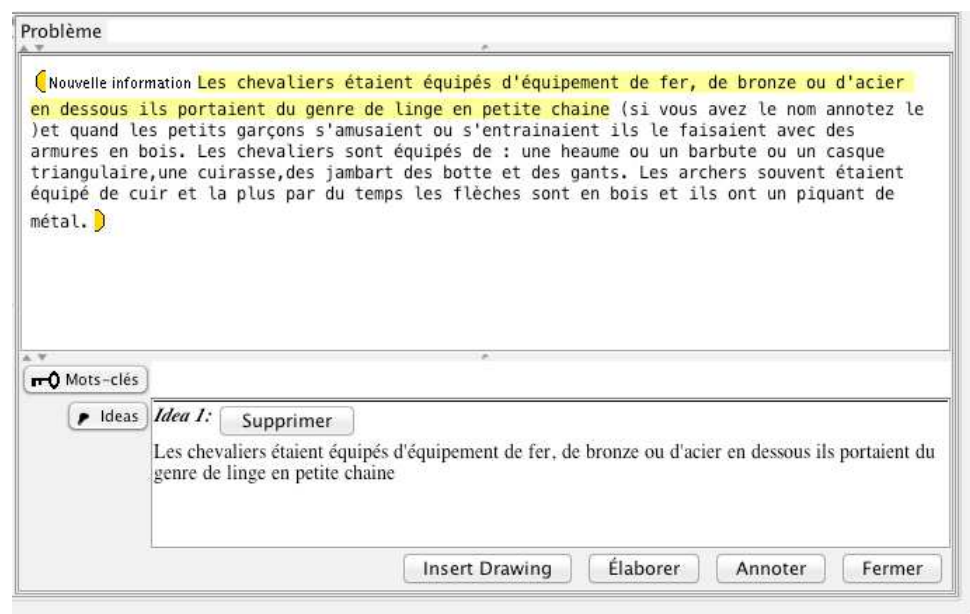

Figure 3 • Surlignement d'une idée prometteuse

Une fois que l'ensemble des élèves eut accompli cela, les enseignantes ont généré en groupe le portrait d'ensemble des idées les plus fréquemment sélectionnées (Figure 4).

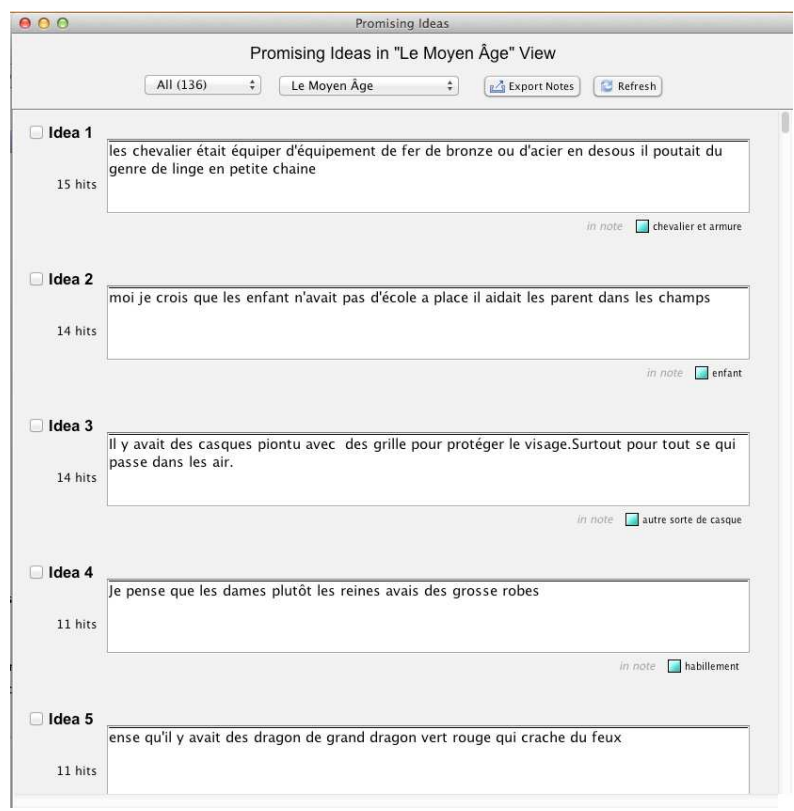

Figure 4 • Portrait des idées prometteuses les plus sélectionnées par les élèves 
À la suite d'une discussion collective, ce portrait a mené à l'identification de 11 thématiques, qui ont donné lieu à la création d'autant de perspectives spécifiques permettant d'orienter la nouvelle phase d'écriture associée à la poursuite de l'investigation du Moyen Âge (Figure 5).

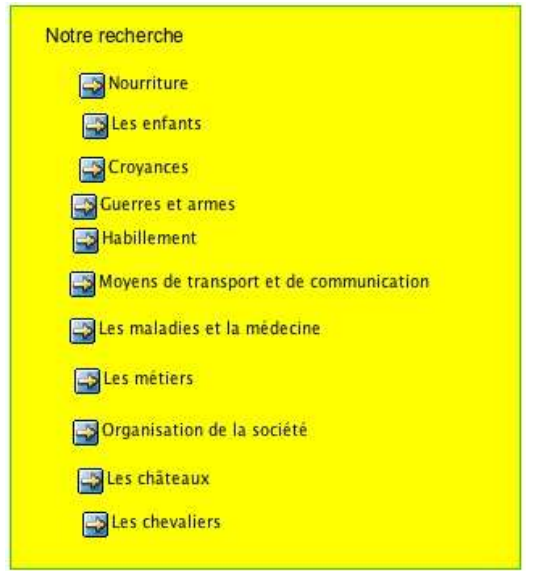

Figure 5 • Perspectives associées aux thématiques retenues par les élèves à l'aide de l'affordance des idées prometteuses à des fins d'investigation du

$$
\text { Moyen Âge }
$$

Les enseignantes ont ensuite insisté sur l'importance de la consultation de ressources documentaires (site web et livres). Elles en ont aussi profité pour enseigner de façon explicite certaines stratégies de repérage et de traitement de l'information de base afin que les élèves puissent reformuler dans leurs propres mots, sur le forum électronique, les informations consultées. L'affordance d'élaboration a été rappelée de sorte à encourager les élèves à construire à partir des idées apportées par les autres. En outre, l'indication des sources a aussi été enseignée pour que les élèves puissent les indiquer dans leurs notes (Figure 6).

(Nouvelle information Au début les chăteaux étaient construits de bois avec des mottes de terres. Plus tard, les châteaux sont construits Source: Les chateau Fort p.6 (Éditions Fleurus))

Figure 6 • Exemple de note rédigée par un élève 
Stéphane ALLAIRE, Pascale THÉRIAULT, Vincent GAGNON, Thérèse LAFERRIÈre, Christine HAMEL, Pier-Ann BOUTIN, Godelieve DEBEURME.

Périodiquement au cours de cette deuxième phase d'écriture, les enseignantes ont projeté au TBI certaines notes d'élèves afin de les amener, en groupe, à réfléchir à des modifications possibles lorsque le contenu semblait plutôt bien ou moins bien correspondre à l'intention d'écriture de la perspective (thématique) ou qu'il manquait de clarté. Des modifications pouvaient être apportées à la note à partir de l'affordance de révision.

Par ailleurs, un étayage a aussi été fourni par les enseignantes sur les perspectives mêmes du forum électronique. Pour ce faire, elles ont utilisé l'affordance d'annotation pour formuler, au total, 33 rétroactions. Ces dernières revêtaient un caractère individuel au sens où elles ciblaient, la plupart du temps, les auteurs de certaines notes de façon spécifique. La majorité des rétroactions fournies $(70 \%)$ ont porté sur le processus d'élaboration de connaissances. Elles ont amené les élèves à préciser la source de leurs informations, à étoffer des contenus, à travailler d'une certaine manière, etc. En voici quelques exemples : « Dans quelles sources as-tu vérifié ? ». «Au lieu d'écrire une élaboration, vous auriez pu faire une annotation. N'oubliez pas de lire ce qui vient avant. Il faut être constructif !». " Il est souhaitable de ne pas répéter ce que les autres ont déjà dit. ». En outre, bien qu'elles aient été nettement moindres, les rétroactions à propos des conventions linguistiques viennent au second rang avec $12 \%$. Quant aux rétroactions concernant la dimension affective (9 \% ), elles ont référé à des encouragements et à des félicitations fournies par les enseignantes en réponse à une note qu'elles ont jugée de qualité. Enfin, les rétroactions concernant la syntaxe ont aussi représenté $9 \%$ de l'ensemble de celles effectuées par les enseignantes à partir de l'affordance d'annotation du Knowledge Forum.

Un étayage par les pairs a aussi été remarqué, c'est-à-dire que les élèves ont également utilisé l'affordance d'annotation en guise de rétroactions aux notes des uns et des autres. Parmi les quelque 441 codes recensés dans les 387 annotations rédigées par les élèves, $83 \%$ étaient de l’ordre du processus d'élaboration comparativement à $17 \%$ pour la dimension affective. Les rétroactions sur le processus ont été diversifiées : ajout de contenu; manifestation d'accord ou de désaccord à propos d'une information; partage ou invitation à partager une source ; demande de clarification de contenu ; confirmation de la qualité d'une information. 


\subsection{Phase 3 (durée approximative : une semaine)}

La troisième phase peut être qualifiée de phase transitoire entre l'écriture collective effectuée sur le Knowledge Forum et une écriture individuelle. Les enseignantes ont invité les élèves à relire le contenu élaboré sur le forum électronique en mettant l'accent sur l'identification de trois idées dont il fallait rendre compte dans un texte informatif individuel. Il s'agissait donc d'une phase d'exploration et de choix d'idées en prévision d'un nouveau contexte d'écriture. Les élèves ont pu s'aider d'un calepin pour noter, en vrac, les idées retenues. À la suite de cette prise de notes, l'organisation du texte individuel a été envisagée. Pour ce faire, les enseignantes ont animé une discussion de groupes en visiocommunication au cours de laquelle elles ont amené les élèves à dégager les principales parties d'un texte informatif. Par la suite, l'importance d'effectuer un plan, préalablement à la mise en texte, a été expliquée et illustrée par un exemple. Chaque élève a ensuite élaboré le plan de son texte.

\subsection{Phase 4 (deux à trois semaines)}

La quatrième phase a consisté en la mise en texte, la révision et la correction du texte individuel. À partir de cette phase, le Knowledge Forum n'a plus été utilisé. Les élèves ont surtout travaillé de façon individuelle, en rédigeant d'abord un brouillon dans le format papier-crayon. Ils ont été soutenus par leur enseignante, par exemple en ce qui a trait à l'enchainement des idées au sein des paragraphes, ainsi que pour la correction linguistique. Lors de la rédaction de cette première version du texte, pour soutenir le regroupement d'idées, un code de couleurs a été proposé par les enseignantes et utilisé par les élèves. Auprès des élèves en difficulté d'apprentissage, un système de jetons a été mis à contribution afin de les amener à intégrer systématiquement certaines étapes de vérification. Par exemple, les élèves devaient placer un jeton sur le verbe après en avoir vérifié l'accord avec le sujet. Peu d'interventions enseignantes ont été observées en lien direct avec la révision du texte ; l'accent a surtout été mis sur la phase de correction des erreurs linguistiques. En complément à cela, la version finale du texte ayant été retranscrite dans Microsoft Word, les outils de correction intégrés à ce traitement de texte ont été utilisés. Lors de la phase de retranscription (du brouillon papier à la version numérique), on a remarqué, d'une part, que des élèves ont continué à modifier le contenu de leur texte ; d'autre part que des erreurs linguistiques se sont ajoutées, possiblement par inattention puisque 
Stéphane ALLAIRE, Pascale THÉRIAULT, Vincent GAGNON, Thérèse LAFERRIÈRE, Christine HAMEL, Pier-Ann BOUTIN, Godelieve DEBEURME.

l'analyse de certains brouillons a révélé que ces erreurs avaient pourtant été corrigées.

\subsection{Phase 5 (durée approximative : une semaine)}

La cinquième phase de la situation d'écriture a consisté en la diffusion de la version finale du texte des élèves (artéfact). Ces textes ont été téléversés sur le site web de l'école à des fins de partage public.

À partir de la description du déroulement de l'ensemble de la situation d'écriture, il a été possible de dégager le modèle de la Figure 7. Ce modèle est l'aboutissement de la conceptualisation effectuée par les chercheurs à partir de ce qui a été planifié et mis en œuvre par les enseignantes.

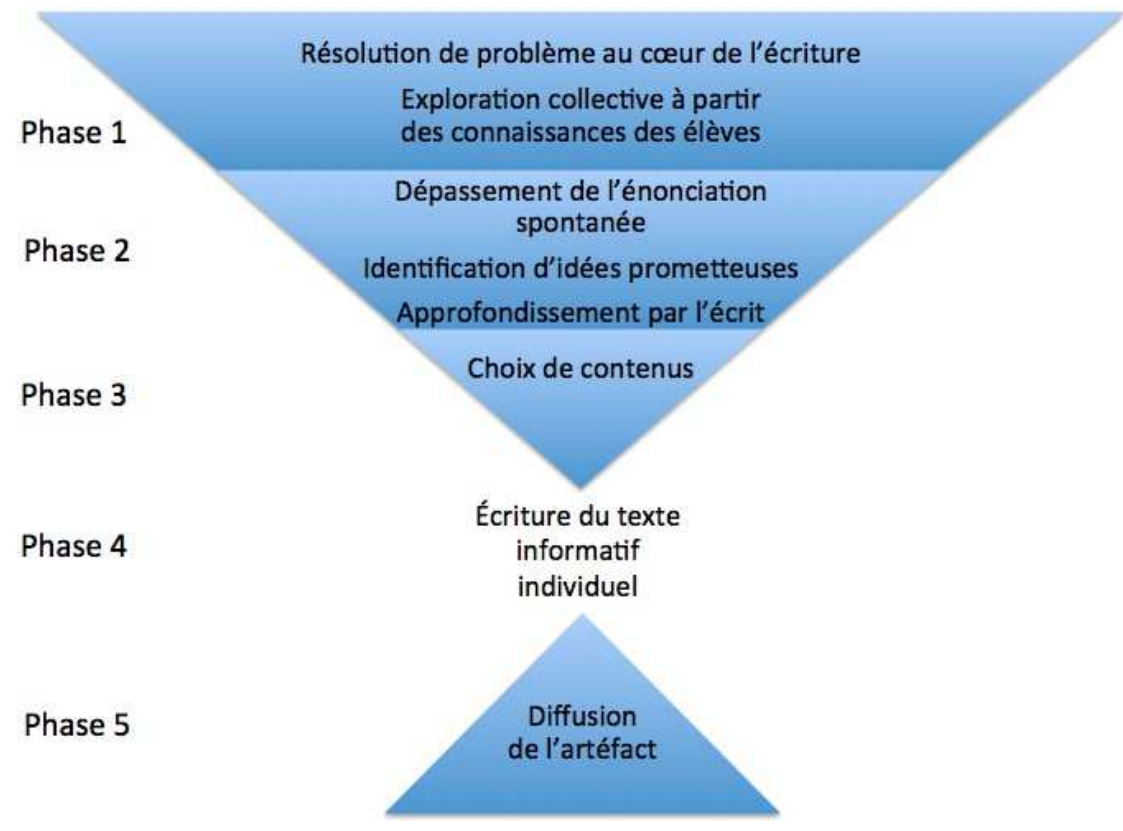

Figure 7 • Modèle dégagé à partir de la situation d'écriture

\subsection{Interventions enseignantes et stratégies d'écriture}

En outre, tout au long de la situation d'écriture, particulièrement lors des trois premières phases, une complémentarité s'est établie entre des interventions enseignantes, des stratégies d'écriture telles qu'elles sont présentées dans le Tableau 3 et des affordances perceptibles du Knowledge Forum (Tableau 3). 
Sticef, Numéro Spécial CREN - Recueil 2013

\begin{tabular}{|c|c|c|c|}
\hline Phases & Interventions enseignantes & Processus et stratégies d'écriture en jeu & $\begin{array}{l}\text { Affordances du Knowledge } \\
\text { Forum mises à contribution }\end{array}$ \\
\hline \multirow{4}{*}{ Phase 1} & $\begin{array}{l}\text { Mise en place d'une situation de résolution de problè- } \\
\text { me : comprendre le fonctionnement de la société au } \\
\text { Moyen Âge et en rendre compte dans un texte infor- } \\
\text { matif }\end{array}$ & $\begin{array}{l}\text { Planification } \\
\text { Utiliser un déclencheur } \\
\text { Préciser une intention d'écriture }\end{array}$ & $\begin{array}{l}\text { Nouvelle note } \\
\text { Échafaudages }\end{array}$ \\
\hline & $\begin{array}{l}\text { Animation d'une discussion en groupe pour faire } \\
\text { émerger de premières idées (tempête d'idées) et leur } \\
\text { consignation sur le Knowledge Forum }\end{array}$ & $\begin{array}{l}\text { Planification } \\
\text { Évoquer un contenu possible } \\
\text { Préciser une intention d'écriture } \\
\text { Mise en texte } \\
\text { Rédiger une première version } \\
\end{array}$ & $\begin{array}{l}\text { Élaboration } \\
\text { Échafaudages }\end{array}$ \\
\hline & $\begin{array}{l}\text { Sensibilisation quant à l'importance de limiter la } \\
\text { redondance des idées }\end{array}$ & $\begin{array}{l}\text { Planification } \\
\text { Choisir des idées }\end{array}$ & $\begin{array}{l}\text { Organisation spatiale et } \\
\text { visuelle de la perspective }\end{array}$ \\
\hline & Explication de la sélection d'idées à approfondir & $\begin{array}{l}\text { Planification } \\
\text { Choisir des idées } \\
\text { Préciser une intention d'écriture } \\
\text { Anticiper l'organisation du texte } \\
\text { Mise en texte } \\
\text { Retourner aux données du projet d'écriture }\end{array}$ & $\begin{array}{l}\text { Idées prometteuses } \\
\text { Perspectives }\end{array}$ \\
\hline \multirow[t]{2}{*}{ Phase 2} & $\begin{array}{l}\text { Explication de stratégies de consultation et de lecture } \\
\text { de ressources documentaires ainsi que de reformula- } \\
\text { tion des propos afin d'alimenter l'approfondissement } \\
\text { des idées prometteuses identifiées }\end{array}$ & $\begin{array}{l}\text { Mise en texte } \\
\text { Relire la partie rédigée pour enchainer la } \\
\text { suite }\end{array}$ & $\begin{array}{l}\text { Élaboration } \\
\text { Échafaudages }\end{array}$ \\
\hline & $\begin{array}{l}\text { Lecture de notes en groupe au TBI et invitation à en } \\
\text { clarifier le contenu }\end{array}$ & $\begin{array}{l}\text { Révision et correction } \\
\text { Lire oralement son texte ou le faire lire afin } \\
\text { d'obtenir des suggestions d'amélioration }\end{array}$ & Révision \\
\hline Phase 3 & $\begin{array}{l}\text { Invitation à relire le contenu élaboré sur le Knowledge } \\
\text { Forum afin d'identifier trois idées principales à déve- } \\
\text { lopper dans le texte individuel }\end{array}$ & $\begin{array}{l}\text { Planification } \\
\text { Exploration et choix d'idées }\end{array}$ & Notes \\
\hline
\end{tabular}

Tableau 3 • Complémentarité entre des interventions enseignantes en regard des stratégies d'écriture et des affordances perceptibles du Knowledge Forum 


\section{Stéphane ALLAIRE, Pascale THÉRIAULT, Vincent GAGNON, Thérèse LAFERRIÈRE, Christine HAMEL, Pier-Ann BOUTIN, Godelieve DEBEURME.}

\subsection{Usage des idées élaborées sur le forum électronique}

Par ailleurs, afin de déterminer si la situation d'écriture élaborée et mise en œuvre par les enseignantes a eu une incidence sur la transformation des connaissances des élèves, une comparaison a été effectuée entre les idées développées sur le Knowledge Forum et celles du texte individuel. Nous cherchions à savoir si le contenu du forum électronique, et le cas échéant quel contenu plus exactement, avait pu inspirer les élèves lors de la rédaction de ce dernier texte. Une analyse effectuée à partir du test non paramétrique de Wilcoxon a révélé que lors de la rédaction du texte individuel, les élèves $(n=34)$ se sont significativement plus inspirés des idées élaborées lors de la phase 2 de la situation d'écriture que de celles de la phase $1(Z=-4.029, p=0.000)$. Cela suggère que les élèves ont transformé leurs représentations spontanées initiales à propos du fonctionnement du Moyen Âge à la lumière des connaissances élaborées par l'écrit. En outre, parmi les idées provenant de la phase 2 , les élèves se sont inspirés davantage de celles provenant de leurs camarades que des leurs $(Z=-2.485$, $\mathrm{p}=0.007)$. À titre indicatif, l'encadré ci-après fournit un exemple de texte individuel d'élève. Le contenu surligné est celui qui provient de la phase 2 du forum électronique.

\section{Une vielle et belle époque}

Sais-tu quelles merveilleuses époques il y avait avant que tu naisses? Une de ces époques était le Moyen Âge, une vieille et chevaleresque époque. Alors, je vais te parler de trois sujets du Moyen Âge : les châteaux, les armes et les métiers.

Les châteaux :

Au Moyen Âge, les premiers châteaux ont été faits en bois érigés sur une butte et entourés d'un profond fossé. Avec le temps, les seigneurs ont voulu améliorer les châteaux avec les taxes payées par leurs vassaux. Les châteaux de pierre ont pris 20 ans de construction. Ils étaient mieux fortifiés et entourés d'un profond fossé. Les armes :

Au Moyen Âge, les armes étaient populaires. L'arc était l'arme la plus facile à fabriquer mais les seigneurs demandaient beaucoup de pratique pour pouvoir combattre avec cette arme. Ensuite, il y avait le bouclier qui était l'arme qui protégeait le mieux. Les premiers étaient faits en bois qu'on devait tenir à deux mains. Ensuite, ils ont évolué pour que les guerriers puissent les tenir à une main et ils sont devenus en fer.

Les métiers :

Au Moyen Âge, il y avait plein de métiers. Par exemple, le métier de forgeron qui consistait à fabriquer des choses de métal. Il y avait aussi le métier de tisserand qui consistait à filer et à tisser ce que les paysans et les marchands portaient. Puis il en avait plein d'autres! 
On remarque qu'aucune idée provenant du temps 1 n'a été retenue, ce qui suggère que l'élève a pris une distance par rapport à ses idées initiales spontanées. Par ailleurs, la provenance des autres idées n'a pu être identifiée, ce qui laisse supposer que les élèves ont poursuivi leur recherche d'idées pendant la phase de rédaction du texte individuel.

Enfin, des idées différentes de celles élaborées sur le Knowledge Forum ont été repérées dans les textes individuels. Il a toutefois été difficile d'identifier leur provenance. La nature du contenu suggère que ces idées proviendraient de ressources documentaires.

\section{Discussion}

Les résultats de cette étude mettent en lumière l'importance des interventions enseignantes en regard de la planification réfléchie mais flexible et de la mise en œuvre d'une situation d'écriture voulant amener les élèves à prendre part à une écriture plus itérative. Les interventions ont tiré profit du design de l'outil technologique retenu. Il n'est pas évident de distinguer précisément les situations où les enseignantes ont instrumentalisé le forum électronique de celles où les affordances de ce dernier ont instrumenté les pratiques. Néanmoins, la situation d'écriture étudiée met en lumière la complémentarité productive et souhaitable qui peut s'instaurer entre la pédagogie et la technologie (Hattie, 2009), (Scardamalia et Bereiter, 1996) lorsqu'un savoir pratique avéré se combine à l'utilisation d'une technologie qui s'appuie sur un rationnel théorique sous-jacent à sa conception. Cela interpelle un enjeu pédagogique important, celui de l'atteinte d'un équilibre entre la guidance offerte et la responsabilisation progressive des élèves.

En ce qui a trait aux processus et aux stratégies d'écriture, lorsqu'on positionne l'usage effectué du forum électronique à travers l'ensemble de la situation d'écriture, on remarque que cette utilisation s'est avérée un soutien majeur à la planification de l'écriture du texte informatif individuel. Le travail effectué sur le Knowledge Forum a non seulement permis d'identifier des thématiques potentielles à traiter dans le texte individuel, mais aussi d'approfondir des idées à y insérer. Il s'agit d'aspects de l'écriture avec lesquels les élèves éprouvent généralement de la difficulté et qui font en sorte qu'ils ont tendance à adopter une stratégie de récitation de connaissances (Bereiter et Scardamalia, 1987). Il apparait qu'une dimension transformative de l'écriture - principalement sur le plan des contenus - a pu prendre forme et qu'elle a été soutenue par cinq principaux éléments : a) l'ancrage de l'écriture à travers une situation de com- 
préhension collective; b) le soutien des enseignantes, qui ont offert un contexte d'écriture d'une flexibilité cadrée ; c) l'interaction avec les pairs, qui ont en quelque sorte stimulé un effet de relance réciproque;d) l'affordance d'élaboration qui a induit un arrimage des idées individuelles à celles des autres; e) la place importante accordée à la lecture des propos des camarades de classe et de ressources documentaires en lien avec l'objet d'investigation.

La situation d'écriture a amené les élèves à composer avec différents niveaux d'intention d'écriture. Un premier niveau était celui de l'objet général de la situation d'écriture (la compréhension de la société au Moyen Âge), qui s'est décliné en plusieurs intentions secondaires de groupe sur le Knowledge Forum, c'est-à-dire les 11 thématiques (perspectives) qui ont émergé à la suite de l'identification des idées prometteuses. En amont de cela, il y avait eu une intention plus individuelle, soit celle de conserver des traces des premières connaissances des élèves. Deux autres niveaux d'intention d'écriture sont entrés en jeu tout au long de la seconde phase d'écriture. En effet, les élèves ne devaient pas uniquement s'assurer que leurs notes étaient en lien avec l'objet général et les thématiques ; ils ont dû aussi identifier la nature du contenu élaboré ainsi que le positionner par rapport à celui déjà présent afin de contribuer à une certaine progression du discours collectif. Lors de la quatrième phase de la situation d'écriture, celle de la rédaction du texte individuel, un dernier niveau d'intention d'écriture a été convoqué, celui de l'information d'un destinataire public, où les élèves ont davantage travaillé la fonction de communication (de ce qu'ils avaient appris) de l'écriture.

Ces divers niveaux d'intention d'écriture nous amènent à effectuer deux constats. D'une part, ils illustrent la dimension foncièrement sociale de la dynamique d'écriture qui a pris forme (Prior, 2006). Les élèves ont écrit à partir, pour et avec d'autres individus. D'autre part, les niveaux d'intention d'écriture mettent en lumière le passage d'une écriture authentique privée à publique, au fur et à mesure que le produit d'écriture des élèves a été réifié. Ainsi, le Knowledge Forum a permis aux élèves d'expérimenter, entre eux, en toute sécurité, le caractère émergent - et donc nécessairement imparfait - de l'écriture, alors que la cinquième phase de la situation d'écriture, celle de diffusion, voulait surtout offrir une vitrine élargie à leur production finie. On peut établir un lien de proximité avec le fonctionnement d'une équipe de recherche, dont le travail d'ébauche et d'élaboration s'effectue d'abord à l'interne, pour plus 
tard être rendu disponible à plus grande échelle, lorsque l'équipe le juge pertinent.

Une caractéristique importante du contexte d'écriture flexible et encadrante nous semble résider dans l'agencement d'une diversité de cycles d'écriture qui ont amené les élèves, d'une façon ou d'une autre, à revisiter leur écriture. Lors des deux premières phases, ce fut davantage sur le plan des idées alors que, tout en demeurant présents lors de la phase 4, les changements apportés aux idées ont laissé place à un travail accru sur la syntaxe et les conventions linguistiques. Globalement, ces différents mouvements rendent compte de l'aspect plus dynamique que statique de l'écriture et, compte tenu que des modifications ont continué à être apportées au texte même lors de la transcription de la version finale, il y a lieu de penser que les élèves ont vécu cette dimension itérative, transformative de l'écriture. Cela dit, l'insertion d'erreurs linguistiques à ce stade soulève des questions quant à la nécessité de combiner les types d'écriture papier et numérique au sein d'une même situation d'écriture.

En ce qui a trait à l'affordance d'élaboration, nous y voyons un moyen de travailler le processus de mise en texte de façon collective avec les élèves, tout particulièrement l'enchainement logique et la progression des idées. Pour préparer un éventuel réinvestissement dans le cadre d'une situation d'écriture individuelle, où l'utilisation de mots-liens et de marqueurs de relation est importante pour assurer cette progression, l'affordance d'échafaudage pourrait être mise à contribution conjointement avec celle d'élaboration. Par exemple, des échafaudages pourraient être proposés aux élèves afin de les amener à positionner explicitement leurs idées par rapport à celles des autres, dans une optique d'enchainement syntaxique. Il nous semble y avoir là une piste à approfondir qui permettrait aux enseignants de travailler concrètement la mise en texte, un processus souvent négligé.

Par ailleurs, certaines affordances n'ont pas été perçues alors qu'elles recèlent de notre point de vue un potentiel intéressant pour soutenir des stratégies d'écriture ainsi qu'une écriture transformative. Nous pensons à l'affordance de citation, qui aurait pu aider à préciser l'ancrage des élaborations, si les notes avaient été d'une longueur accrue. Nous pensons aussi au champ " Problème », qui pourrait permettre aux élèves de se remémorer le questionnement initial à chaque note rédigée. Enfin, nous pensons à l'affordance de la note "Élever le propos ", qui, elle, aurait pu être utilisée par différentes équipes d'élèves pour rédiger un brouillon collectif en 

DEBEURME.

lien avec chacune des 11 perspectives retenues portant sur le Moyen Âge. L'utilisation de cette affordance aurait pu permettre d'intégrer un cycle d'écriture supplémentaire à la situation globale.

En guise de conclusion, cette étude a permis d'illustrer qu'il est possible de travailler une écriture plus itérative avec des élèves du primaire. Qui plus est, ceux qui ont participé à l'étude provenaient d'un milieu socioéconomique défavorisé. La situation d'écriture analysée en fut une d'intégration de matières scolaires. La dimension collective de l'écriture ressort comme un levier pertinent à exploiter. En outre, la complémentarité qui s'est établie entre les interventions enseignantes et les affordances du forum électronique utilisé invite à une réflexion minutieuse à propos du type de soutien offert aux élèves et du design des outils technologiques. Nos résultats mettent en exergue l'importance, pour les enseignants, de posséder une connaissance fine de la démarche d'écriture. Une piste de recherche éventuelle pourrait consister à documenter si les résultats obtenus peuvent se transposer lorsque d'autres outils d'écriture sont mis à profit (blogue, wiki, etc.). Enfin, nous soulevons une tension qui nous semble nécessaire d'approfondir. Du point de vue spécifique de l'apprentissage individuel de l'écriture, la démarche qui émerge des analyses effectuées tend à soutenir le passage d'un niveau interpsychologique à un niveau intrapsychologique (Vygotsky, 1978). Toutefois, elle pose la question de la production d'un artéfact collectif, qui elle caractérise la dynamique de coélaboration de connaissances. L'arrimage de ces deux éléments demeure un questionnement, tant du point de vue de la recherche que de l'intervention.

\footnotetext{
${ }^{1}$ Les deux autres types recensés sont les pratiques scriptées (fortement dirigées) et de laisser-aller. La première réfère à des situations d'écriture dont les consignes sont particulièrement directives alors que la seconde réfère à des situations où l'enseignant n'intervient à peu près pas. Les trois types ont émergé au fil de l'analyse de l'ensemble des situations d'écriture mises en œuvre dans le cadre de la recherche.
}

\section{BIBLIOGRAPHIE}

ALLAIRE S. (2006). Les affordances socionumériques d'un environnement d'apprentissage hybride en soutien à des stagiaires en enseignement secondaire. De l'analyse réflexive à la coélaboration de connaissances. Université Laval, Québec.

ALLAIRE S., LUSIGNAN G. (2011). Enseigner et apprendre en réseau: collaborer entre classes distantes à l'aide des TIC. Anjou: Éditions CEC.

ALLAIRE S., THERIAULT P., LAFERRIERE T., HAMEL C., DEBEURME G. (2013). Écrire ensemble au primaire à l'aide du Forum de coélaboration de connaissances: interventions d'enseignants et stratégies d'écriture des élèves. Rencontre de suivi au Fonds de recherche québécois sur la société et la culture, Québec. 
ALLAIRE S., THERIAULT P., GAGNON V., NORMANDEAU L. (2013). Étude de cas multiples sur le développement de l'écriture dans des classes du secondaire utilisant le blogue. Rapport de recherche présenté au Ministère de l'Éducation, du Loisir et du Sport. Saguenay: Université du Québec à Chicoutimi. 106 pages. [En ligne]. Disponible http://constellation.uqac.ca/2450/

ALLAIRE S., THERIAUlT P., GAGNON V., LALANCETTE E. (2011). Environnements d'apprentissage intégrant le blogue au primaire : de la dimension affective à la dimension cognitive de l'écriture. Rapport de recherche présente au Ministère de l'Éducation, du Loisir et du Sport. Saguenay: Université du Québec à Chicoutimi. 90 pages.

BEREITER C. (2002). Education and mind in the knowledge age. Mahwah: Lawrence Erlbaum Associates.

BEREITER C., SCARDAMALIA M. (2005). Technology and literacies: From print literacy to dialogic literacy. In N. Bascia, A. Cumming, A. Datnow, K. Leithwood, et D. Livingstone (Eds.), International handbook of educational policy (p. 749-761). Dordrecht, Netherlands: Springer.

BEREITER C., SCARDAMALIA M. (2003). Learning to work creatively with knowledge. In E. De Corte, L. Verschaffel, N. Entwistle et J. van Merrienboer (Eds.), Powerful learning environments: Unravelling basic components and dimensions (p. 5568). Oxford UK: Pergamon.

BEREITER C., SCARDAMALIA M. (1987). The Psychology of Written Composition. Hillsdale, Ney Jersey : Erlbaum.

BLASER C. (2007). Fonction épistémique de l'écrit: pratiques et conceptions d'enseignants de sciences et d'histoire du secondaire. Université Laval, Québec.

BOUTIN P-A. (2013). Processus d'amélioration des idées et discours collectif d'élèves du primaire: l'identification des idées prometteuses et ses suites. Université Laval: Québec.

ENGESTRÖM Y. (1987). Learning by expanding: An activity-theoretical approach to developmental research. Helsinki, Finland: Orienta-Konsultit.

GAVER W. (1991). Technology affordances. CHI, New Orleans.

GIBSON J. (1979). The theory of affordances. In R. Shaw et J. Bransford (Eds.), Perceiving, Acting and Knowing. Hillsdale: Erlbaum.

HATTIE J. (2009). Visible learning. A synthesis of over 800 meta-analyses relating to achievement. New York: Routledge.

HAYES J. (1995). Un nouveau modèle du processus d'écriture. In J.Y. Boyer, J.P. Dionne et P. Raymond (Dir.), La production de textes : vers un modèle d'enseignement de l'écriture (p. 49-72), Montréal : Éditions Logiques.

HAYES J., FLOWER S. (1980). Identifying the organization of writing processes. In. L.W. Gregg et E.R. Steinberg (Eds.), Cognitive processes in writing (p. 3-30), Hillsdale: Lawrence Erlbaum.

LAFERRIERE T., HAMEL C., ALLAIRE S., TURCOTTE S., BREULEUX A., BEAUDOIN J., et al. (2011). L'École éloignée en réseau, un modèle. Rapport-synthèse, octobre 2011. CEFRIO.

MINISTERE DE L'EDUCATION (2001). Programme de formation de l'école québécoise (Éducation préscolaire et enseignement primaire). Québec: Gouvernement du Québec.

PRIOR P. (2006). A sociocultural theory of writing. In C. MacArthur, S. Graham, et J. Fitzgerald (Eds.), Handbook of writing research (pp. 54-66). New York: Guilford. 
Stéphane ALLAIRE, Pascale THÉRIAULT, Vincent GAGNON, Thérèse LAFERRIÈRE, Christine HAMEL, Pier-Ann BOUTIN, Godelieve DEBEURME.

RABARDEL P. (1995). Les hommes et les technologies. Approche cognitive des instruments contemporains. Paris : Armand Colin.

SCARDAMALIA M., BEREITER C. (2006). Knowledge building: Theory, pedagogy, and technology. In K. Sawyer (Ed.), Cambridge Handbook of the Learning Sciences (p. 97-118). New York: Cambridge University Press.

SCARDAMALIA M., BEREITER C. (1996). Engaging students in a knowledge society. Educational Leadership, Vol. 54 n³, p.6-10.

SCHLEICHER A. (Ed.) (2012). Preparing teachers and developing school leaders for the $21^{\text {st }}$ century: Lessons from around the world. Paris: OCDE.

UNESCO. (2011). Standards de compétence TIC des enseignants. Paris : Auteur.

UNESCO. (2005). Towards Knowledge Societies. Disponible sur internet: http://portal.unesco.org/ci/en/ev.php-

URL ID=20493\&URL DO=DO TOPIC\&URL SECTION=201.html

VYGOTSKY L. S. (1978). Mind in Society. The Development of Higher Psychological Processes. Cambridge MASS et London ENG: Harvard University Press. 